Pacific Journal of Mathematics

MINIMAL RANGE THEOREMS FOR OPERATORS WITH THIN 


\title{
MINIMAL RANGE THEOREMS FOR OPERATORS WITH THIN SPECTRA
}

\author{
JoSEPH G. StAMPfLi
}

Let $T$ be a bounded linear operator on a Hilbert space $H$. Let $W(T)=\{(T x, x): x \in H$ and $\|x\|=1\}$ denote the numerical range of $T$, and let $\Sigma(T)$ designate the convex hull of $\sigma(T)$, the spectrum of $T$. It is well known that for an arbitrary operator $T, \Sigma(T) \subset \overline{W(T)}$. Moreover, if $T$ is normal, then $\overline{W(T)}=\Sigma(T)$. In general, if $\overline{W(T)}=\Sigma(T)$, one can not expect $T$ to be normal. However, if the spectrum of $T$ is sufficiently thin, then relations of this sort do imply something about the operator.

First it is shown that, for operators with spectrum on certain "flat" convex curves, one can infer from the relations $W\left(T^{ \pm 1}\right) \subset \Sigma\left(T^{ \pm 1}\right)$ alone that $T$ is normal. Examples are presented which show that this inference can not be made for arbitrary convex curves. However, the second result states that if $\sigma(T)$ lies on a smooth convex curve, and

(a) $W(T) \subset \Sigma(T)$

(b) $W\left[(T-z I)^{-1}\right] \subset \Sigma\left[(T-z I)^{-1}\right]$ for $z \notin \sigma(T)$, then $T$ is normal.

Many conditions on $T$, short of normality, are known to imply (a) or (b), and corollaries are stated to cover these situations.

The results in this area to date are few. If $W(T)$ lies on a line, then $T$ is obviously a scalar multiple of a self-adjoint operator. Donoghue [2] has shown that if $W(T) \subset\{z:|z| \leqq 1\}$ and ||$T^{-1} \mid \leqq 1$, then $T$ is unitary. The two situations just cited are the cases where $\sigma(T)$ lies on a line or a circle. Meng [4] has considered the case where $\sigma(T)$ lies on a convex curve and $W(T) \subset \Sigma(T)$. He obtains results on the point and residual spectrum of the operator. This appears to be the extent of previous efforts to obtain a converse to the statement that $T$ normal implies $W(T) \subset \Sigma(T)$.

I. We begin with some preliminary material on curves in the complex plane. We shall consider only compact connected curves which may or may not separate the plane. Such a curve is convex if it is contained in the boundary of its convex hull ([10] p. 105). Thus, a curve is convex if and only if it has a support line at every point ([10] p. 47).

We define a curve $C$ to be convex at the point $p \in C$ if it has $\varepsilon$ support line at $p$. Under this definition, a cusp could be a convex point, which is disquieting. However, when we use this definition 
later in $\S I I$, we shall consider only the case where a curve is convex at every point (on the curve) in a neighborhood, and then the definition agrees with intuition.

Let $C$ be a convex curve. If, at every point $p \in C$, there exists a support line such that the associated closed half plane which contains $C$ also contains the origin, then we say $C$ is convex with respect to the origin. (It is explicitly assumed that the curve does not contain the origin.)

The curve $C \in \mathscr{C}$, if

(1) $C$ is given, in polar form, by a single valued continuous function $R=f(\theta)$, where $R>0$ for $\delta_{1} \leqq \theta \leqq \delta_{2}$,

(2) both $C$ and $C^{-1}(R=1 / f(-\theta))$ are convex,

(3) both $C$ and $C^{-1}$ are convex with respect to the origin.

We will need a few elementary properties of curves in $\mathscr{C}$. The proofs are not hard, but we sketch them for completeness. Note that the definition of $\mathscr{C}$ is symmetric, that is, if $C \in \mathscr{C}$ then $C^{-1} \in \mathscr{C}$. It follows from the continuity of $f(\theta)$ that $C$ is compact. Even if we did not assume $f(\theta)$ to be continuous, the other hypotheses on $C \in \mathscr{C}$ imply that it is. (For continuity at an end point $\delta_{i}$, the symmetry of $\mathscr{C}$ is crucial.) A curve $C \in \mathscr{C}$ may or may not separate the plane.

Lemma 1. Let $p \in C \in \mathscr{C}$. Then $C$ has a tangent at $p$; that is, the support line to $C$ at $p$ is unique, if $p$ is not an end point.

Proof. Since $C$ is convex, the right and left hand tangents at $p$ exist. The only difficulty is the possibility of a "corner" at $p$. However, consider a support line $L$ to $p^{-1}$ at $C^{-1}$. Then $L^{-1}$ is a circle passing through $p$ and the origin, which lies between the origin and $C$. Thus, it is not possible for $C$ to have a corner at $p$.

For $R_{0} e^{i \theta_{0}}$ on $C \in \mathscr{C}$, consider the angle between the ray $r e^{i \theta_{0}}$ and the tangent line $S$ to $C$ at this point. Set $A\left(\theta_{0}\right)$ equal to the angle between $S$ and $r e^{i \theta_{0}}$, measured in the counter-clockwise sense. Thus, $A(\theta)$ is defined for $\delta_{1} \leqq \theta \leqq \delta_{2}$, where $0 \leqq A(\theta) \leqq \pi$ and the ambiguity at 0 and $\pi$ will not be a problem. In fact, the next lemma rules out such occurrences.

Lemma 2. Let $C \in \mathscr{C}$. Define

$$
\phi=\sup \left\{|A(\theta)-\pi / 2|: \delta_{1} \leqq \theta \leqq \delta_{2}\right\} .
$$

Then $0 \leqq \phi<\pi / 2$.

Proof. Assume $\phi=\pi / 2$. Then there exist $z_{n} \in \mathscr{C}$, with $z_{n} \rightarrow z_{0}$ and $A\left(\theta_{n}\right) \rightarrow 0$ or $\pi$, where $z_{n}=R_{n} e^{i \theta_{n}}$. Consider the case $A\left(\theta_{n}\right) \rightarrow 0$, 
by selecting a subsequence if necessary. An obvious argument shows that by a further selection, the $z_{n}$ 's must be increasing (as a function of $\theta$ ). We claim $A\left(\theta_{0}\right)=0$. If not, choose $A\left(\theta_{n}\right)<A\left(\theta_{0}\right)$. Then the tangent line $S_{n}$ to $C$ at $z_{n}$ divides the plane into two components with $z_{0}$ in one and the origin in the other, contrary to hypothesis.

Since $A\left(\theta_{0}\right)=0$, for any $\varepsilon$, there exists a line $L_{\varepsilon}$ through $z_{0}$ and $u_{\varepsilon} \in C$ such that $\arg w_{1}<\arg u_{\varepsilon}<\arg z_{0}$, and moreover, the angle between the ray $r e^{i \theta_{0}}$ and $L_{\varepsilon}$ is less than $\varepsilon$. This implies that the angle between the circle $L_{\varepsilon}^{-1}$ and the ray $r e^{-i \theta_{0}}\left(\right.$ at $z_{0}^{-1}$ ) is less than $\varepsilon$. It should be clear that for $\varepsilon$ sufficiently small, the point $u_{\varepsilon}^{-1}$ lines inside the triangle with vertices $z_{0}^{-1}, w_{1}^{-1}, 0$. Let $S$ be the support line to $C^{-1}$ at $u_{\varepsilon}^{-1}$. Since $C^{-1}$ is convex with respect to the origin, $z_{0}^{-1}, w_{1}^{-1}, 0$ all lie on the same side of $S$, which is impossible. The proof is complete.

Lemma 3. Let $\lambda e^{i \theta} \in C$, where $C \in \mathscr{C}$. If $\mu>\lambda$, then distance $\left[\mu e^{i \theta}, C\right]>K_{1}(\mu-\lambda)$, for some $K_{1}>0$, where $K_{1}$ is independent of $\theta$.

Proof. Let $S$ be the tangent line to $C$ at $\lambda e^{i \theta}$. Then

$$
\begin{aligned}
\operatorname{dist}\left[\mu e^{i \theta}, C\right] \geqq \operatorname{dist}\left[\mu e^{i \theta}, S\right] & =(\mu-\lambda) \sin A(\theta) \\
& =(\mu-\lambda) \cos [A(\theta)-\pi / 2] \geqq(\mu-\lambda) \cos \phi
\end{aligned}
$$

which completes the proof, since $0 \leqq \phi<\pi / 2$ and $\phi$ is independent of $\theta$.

We are now ready to state the first of our main results.

THEOREM 1. Let $\sigma(T)$ lie on a curve $C \in \mathscr{C}$. Then $T$ is normal if and only if $W\left(T^{ \pm 1}\right) \subset \Sigma\left(T^{ \pm 1}\right)$.

Proof. Assume $W\left(T^{ \pm 1}\right) \subset \Sigma\left(T^{ \pm 1}\right)$. Let $T=B U$ be the polar decomposition of $T$. Since $T$ is invertible, $U$ is unitary and $B$ is invertible. Let $U=\int e^{2 \pi i t} d E(t)$. We next divide the circle into $N$ equal parts, and define projections $P_{k}=E([(k-1) / N, k / N])$ for $k=1, \cdots, N$. For $x \in P_{k} H$, set $U x=a x+b y$, where $(x, y)=0$ and $\|x\|=\|y\|=1$. Thus $|a|^{2}+|b|^{2}=1$. But

$$
a=(U x, x)=\int_{(k-1) / N}^{(k / N)-0} e^{2 \pi i t} d\|E(t) x\|^{2},
$$

hence $a$ lies in the convex hull of the arc $\left[e^{2 \pi i(k-1) / N}, e^{2 \pi i k / N}\right]$ and so $1-|a| \leqq \pi^{2} / 2 N^{2}$. This ingenious observation is due to Donoghue [2] and the order $N^{-2}$ is crucial to the proof. It is immediate that $|b| \leqq \pi / N$.

For $x \in P_{k} H$, set $B x=\lambda x+\beta w$, where again $\|x\|=\|w\|=1$ 
and $(x, w)=0$. We will now show that $\beta$ is $O(1 / N)$. For $x \in P_{k} H$, note that

$$
(T x, x)=(U x, B x)=(a x+b y, \lambda x+\beta w)=a \lambda+b \bar{\beta}(y, w) .
$$

Let $a \lambda_{0}$ be the point on $C$ whose argument is $\arg a$. If $\lambda<\lambda_{0}$, we may take $\lambda=\lambda_{0}$ later, and dispense with the present estimate. If $\lambda \geqq \lambda_{0}$, it follows from Lemma 3 that

$$
\operatorname{dist}[a \lambda, W(T)] \geqq|a|\left(\lambda-\lambda_{0}\right) K_{1},
$$

where $K_{1}>0$. Since $a \lambda+b \bar{\beta}(y, w) \in W(T)$, we conclude that

$$
|a|\left(\lambda-\lambda_{0}\right) K_{1} \leqq|b \beta(y, w)| \leqq|b \beta|,
$$

and thus

$$
|a|\left(\lambda-\lambda_{0}\right) \leqq K_{2}|b \beta|,
$$

where $K_{2}$ is a constant independent of $N$. From here on, all constant $K_{i}$ are independent of $N$. Since $B x=\lambda x+\beta w$, it follows that $B^{-1} x=$ $\lambda^{-1} x-\beta \lambda^{-1} B^{-1} w$. Hence,

$$
\begin{aligned}
\left(T^{-1} x, x\right) & =\left(B^{-1} x, U x\right) \\
& =\left(\lambda^{-1} x-\beta \lambda^{-1} B^{-1} w, a x+b y\right) \\
& =\bar{a} \lambda^{-1}-\beta \lambda^{-1}\left(w, B^{-1}(a x+b y)\right) \\
& =\bar{a} \lambda^{-1}+\bar{a}|\beta|^{2} \lambda^{-2}\left(w, B^{-1} w\right)-\beta \lambda^{-1}\left(w, B^{-1} b y\right) \\
& =\bar{a}\left[\lambda^{-1}+|\beta|^{2} \lambda^{-2}\left(B^{-1} w, w\right)\right]-\beta \lambda^{-1}\left(B^{-1} w, b y\right) .
\end{aligned}
$$

Observe that $\left(a \lambda_{0}\right)^{-1}$ is the point on $C^{-1}$ whose argument is $\arg \bar{a}$. Let $h=\bar{a}\left[\lambda^{-1}+|\beta|^{2} \lambda^{-2}\left(B^{-1} w, w\right)\right]$. We assume $|h| \geqq\left|\left(a \lambda_{0}\right)^{-1}\right|$; if not, the estimate below follows easily and directly. We are in point of fact doing the difficult case. By Lemma 3,

$$
\operatorname{dist}\left[h, W\left(T^{-1}\right)\right] \geqq\left[|h|-\left|\left(a \lambda_{0}\right)^{-1}\right|\right] K_{1} .
$$

Since $h-\beta \lambda^{-1}\left(B^{-1} w, b y\right) \in W\left(T^{-1}\right)$, we see that

$$
\left[|h|-\left|\left(a \lambda_{0}\right)^{-1}\right|\right] K_{1} \leqq\left|\beta \lambda^{-1}\left(B^{-1} w, b y\right)\right|,
$$

and hence

$$
|h|-\left|\left(a \lambda_{0}\right)^{-1}\right| \leqq K_{3}|b \beta|
$$

or equivalently

$$
|a|\left[\left(\lambda^{-1}-\lambda_{0}^{-1}\right)+|\beta|^{2} \lambda^{-2}\left(B^{-1} w, w\right)\right]+\lambda_{0}^{-1}\left(|a|-|a|^{-1}\right) \leqq K_{3}|b \beta| .
$$

Thus, 


$$
\begin{aligned}
|a||\beta|^{2} \lambda^{-2}\|B\|^{-1} & \leqq K_{3}|b \beta|+K_{4}\left[|a|\left|\lambda-\lambda_{0}\right|+\lambda_{0}^{-1} / N^{2}\right] \\
& \leqq K_{3}|b \beta|+K_{5}|b \beta|+K_{5} / N^{2}
\end{aligned}
$$

which implies that

$$
\left(1-\pi^{2} / 2 N^{2}\right)|\beta|^{2} \leqq K_{6}\left(|\beta| / N+1 / N^{2}\right) .
$$

From this, it follows that $|\beta| \leqq K_{7} / N$ for some constant $K_{7}$ which is independent of $N$.

For $x \in P_{k} H$, observe that

$$
B U x=a \lambda x+a \beta w+b B y
$$

and

$$
U B x=a \lambda x+b \lambda y+\beta U w .
$$

Thus,

$$
\|(B U-U B) x\| \leqq|b|[|\lambda|+\|B\|]+|\beta|[|a|+\| U||] \leqq K_{8} / N .
$$

Now we are prepared to prove the theorem. For $u \in H$ with $\|u\|=1$, let $u=\sum_{1}^{N} a_{k} x_{k}$, where $x_{k} \in P_{k} H$. Thus, $\sum_{1}^{N}\left|a_{k}\right|^{2}=1$. Accordingly,

$$
\begin{aligned}
\|(U B-B U) u\| & \leqq \sum_{1}^{N}\left|a_{k}\right|\left\|(U B-B U) x_{k}\right\| \\
& \leqq\left[\sum_{1}^{N}\left|a_{k}\right|^{2} \sum_{1}^{N}\left(K_{8} / N\right)^{2}\right]^{1 / 2} \leqq K_{8} / N^{1 / 2} .
\end{aligned}
$$

Since $N$ is arbitrary, we see that $U B=B U$, hence $T$ is normal.

The other half of the theorem is well known for normal operators.

COROLlaRY 1. If $W\left(T^{ \pm 1}\right) \subset\{|z| \leqq 1\}$, then $T$ is unitary.

Proof. Since $W(T) \subset\{|z| \leqq 1\}$, it follows that $\sigma(T) \subset\{|z| \leqq 1\}$. The same is true for $T^{-1}$, and together these yield $\sigma(T) \subset\{|z|=1\}$. The curve $|z|=1$ is clearly in $\mathscr{C}$. Thus, $W\left(T^{ \pm 1}\right) \subset \Sigma\left(C^{ \pm 1}\right)$ for $C \in \mathscr{C}$ and this is sufficient. See added in proof.

Corollary 2. (Donoghue's Theorem) If $\left\|T^{-1}\right\| \leqq 1$ and $W(T) \subset$ $\{|z| \leqq 1\}$, then $T$ is unitary.

Proof. Certainly $\left\|T^{-1}\right\| \leqq 1$ implies that $W\left(T^{-1}\right) \subset\{|z| \leqq 1\}$. The result now follows from Corollary 1.

Corollary 3. Let $C \in \mathscr{C}$. Assume 


$$
\|(T-z I)\| \leqq 1 / \operatorname{dist}(z, \Sigma(T)) \quad \text { for } z \notin \Sigma(T),
$$

and

$$
\left\|\left(T^{-1}-z I\right)^{-1}\right\| \leqq 1 / \operatorname{dist}\left(z, \Sigma\left(T^{-1}\right)\right) \quad \text { for } z \notin \Sigma\left(T^{-1}\right) .
$$

Then $T$ is normal.

Proof. Orland has shown [6] that $\left\|(T-z I)^{-1}\right\| \leqq 1 / \operatorname{dist}(z, \Sigma(T))$ if and only if $W(T) \subset \Sigma(T)$. Thus the conditions above imply that $W\left(T^{ \pm 1}\right) \subset \Sigma\left(T^{ \pm 1}\right)$, and the conclusion follows.

Actually, we could have made Corollary 3 more elaborate since there are conditions, other than those given, which imply $W(T) \subset \Sigma(T)$ or $W\left(T^{-1}\right) \subset \Sigma\left(T^{-1}\right)$. These are used later in Corollary 1 to Theorem 2, and thus the reader may embellish this corollary himself by following the schema there.

Next comes the question of relaxing the conditions on $\mathscr{C}$. This is complicated by the fact that the conditions are not independent. Thus, if one omits the requirement that $C$ be given (in polar form) by a single-valued function $R=f(\theta)$, then immediately one has ruled out the possibility that $C$ is convex with respect to the origin.

We could give a single example which indicates the necessity of all three conditions on $\mathscr{C}$. However, it seems worthwhile to present two essentially different examples.

First we define

$$
V \phi_{1}=1 / 2 \phi_{2} \quad \text { and } \quad V \dot{\phi}_{i}=\phi_{i+1} \quad \text { for } i \neq 1
$$

where $\left\{\phi_{i}\right\}$ for $i=0, \pm 1, \pm 2, \cdots$ is an orthonormal basis for $H$. Since $V$ is similar to the bilateral shift, $\sigma(V)=\{z:|z|=1\}$. Clearly, $V$ is a contraction. Hence the $\operatorname{disc} D=\{z:|z| \leqq 1\}$ is a spectral set for $V$, and $W(V) \subset D$. Let $T=3 I+V$. Then $W(T) \subset \Sigma(T)$. Now $\sigma\left(T^{-1}\right)$ is a circle, call it $\Gamma$. Since $\{3+D\}$ is a spectral set for $T$, the image of $\{3+D\}$ under the map $\zeta=1 / z$ is a spectral set for $T^{-1}$. The image of $\{3+D\}$ under this map is just the convex hull of $\Gamma$. But the numerical range of an operator is contained in any convex spectral set for the operator [8], hence $W\left(T^{ \pm 1}\right) \subset \Sigma\left(T^{ \pm 1}\right)$. However, $T$ is clearly not a normal operator.

In our next example, we will construct an operator $T$ where

(1) $\sigma(T)$ is given (in polar form) as $R=f(\theta)$,

(2) both $\sigma(T)$ and $\sigma\left(T^{-1}\right)$ are convex curves,

(3) $\sigma\left(T^{-1}\right)$ is convex with respect to the origin, and a reflection of $\sigma(T)$ is convex with respect to the origin.

Condition (3) states that the convexity orientation has flip-flopped, so to speak, under the map $\zeta=1 / z$. Moreover, for this $T, W\left(T^{ \pm 1}\right) \subset$ $\Sigma\left(T^{ \pm 1}\right)$, but $T$ is not normal. 
Let $M$ be the broken line segment which joins $2+3 i$ to $1+i$, $1+i$ to $1-i$ and $1-i$ to $2-3 i$. Clearly, $M$ is given by $R=f(\theta)$ and $M$ and $M^{-1}$ are both convex curves. Furthermore, both $M^{-1}$ and the reflection of $M$ with respect to the line $\operatorname{Re} z=1$ are convex with respect to the origin.

Define a normal operator $N$ such that $\sigma(N)=M$. Let $Q$ be the Volterra operator on $L^{2}[0,1]$, that is, $\{Q f\}(s)=\int_{0}^{s} f(t) d t$. It is well known that $Q$ is quasi-nilpotent and $W(Q)$ lies in the right half plane. Let $R=I+\delta Q$, where $\delta$ will be specified later. We define our operator $T$ as $T=N \oplus R$. It should be clear that $\sigma(T)=M$ and $W(T) \subset \Sigma(T)$ for $\delta$ sufficiently small (actually $\delta \leqq 1$ will do at this stage).

Since $W(T) \subset\{\operatorname{Re} z \geqq 1\}$, the half plane $\operatorname{Re} z \geqq 1$ is a spectral set for $T$. Hence the circle $\{|z-1 / 2| \leqq 1 / 2\}$, which is the image of $\{\operatorname{Re} z \geqq 1\}$ under the map $\zeta=1 / z$, is a spectral set for $T^{-1}$. But the numerical range of an operator is contained in any convex spectral set for the operator, and thus $W\left(T^{-1}\right) \subset\{|z-1 / 2| \leqq 1 / 2\}$. Obviously, $T^{-1}=N^{-1} \oplus R^{-1}$. The contribution to $W\left(T^{-1}\right)$ made by $N^{-1}$ is not going to cause any difficulties. Note that $\left\|I-R^{-1}\right\| \leqq \delta /(1-\delta)$. Hence, $W\left(R^{-1}\right) \subset\{|z-1| \leqq \delta(1-\delta)\}$. This yields

$$
W\left(R^{-1}\right) \subset\{|z-1| \leqq \delta /(1-\delta)\} \cap\{|z-1 / 2| \leqq 1 / 2\} .
$$

Thus for $\delta$ sufficiently small, it should be clear that $W\left(R^{-1}\right) \subset$ convex hull $M^{-1}$. This implies $W\left(T^{-1}\right) \subset \Sigma\left(T^{-1}\right)$ as claimed.

We have therefore produced a nonnormal operator $T$ with all the promised attributes. This example makes clear that the theorem depends not only on the convexity of $\sigma(T)$ and $\sigma\left(T^{-1}\right)$, but also on their convexity orientation being preserved under inversion. If the curve $\sigma(T)$ flips-flops under inversion, there is no hope of obtaining normality without additional conditions.

II. Let $C$ be a simple curve in the complex plane with parametric representation $x=x(t), y=y(t)$ for $0 \leqq t \leqq 1(C$ is permitted to be closed). If $x$ and $y$ have continuous second derivatives, we say that $C$ is smooth. The expression $\left\{C+z_{0}\right\}$ designates the curve $C$ translated by an amount $z_{0}$. Recall that a curve $C$ is convex at the point $p \in C$ if it has a support line at $p$.

Lemma 4. Let $q \in C$, where $C$ is a smooth convex curve. Then there exist $z_{0}, \varepsilon>0$, such that for

$$
w \in\left\{z:\left|z-\left(q-z_{0}\right)^{-1}\right| \leqq \varepsilon\right\} \cap\left[C-z_{0}\right]^{-1},
$$

the curve $\left[C-z_{0}\right]^{-1}$ is convex at $w$. 
Proof. Let $q \in C$ be given. We will assume that the normal to $C$ at $q$ passes through the origin, and $q \neq 0$. The general case requires only obvious modifications.

Since $C$ is smooth, the curvature $K(p)$ is a continuous function of $p$ for $p \in C$. Let $A=\max \{K(p): p \in C\}$. Consider $[C-\rho q]$, and choose $\rho$ real so that $0<|q-\rho q| \leqq 2 / A$. Then the circle through $(q-\rho q)$ and 0 , tangent to $[C-\rho q]$, lies inside $[C-\rho q]$, or between $[C-\rho q]$ and the origin. Moreover, by continuity and the way in which $\rho$ was chosen, for $u \in C$ and $u$ sufficiently near $q$, the circle $\Gamma_{u}$ through $u-\rho q$ and 0 , tangent to $[C-\rho q]$, also lies between $[C-\rho q]$ and the origin. Under the map $\zeta=1 / z$, the circle $\Gamma_{u}$ goes into the tangent $L_{u}$ to $[C-\rho q]^{-1}$ at $(u-\rho q)^{-1}$. But, since $\Gamma_{u}$ lies inside $[C-\rho q]$, its image, the tangent $L_{u}$, lies on one side of $[C-\rho q]^{-1}$, and hence is the support line we promised.

Using this lemma, we obtain the following analogue to Lemma 3, even though $\left[C-z_{0}\right]^{-1}$ is not convex in general.

Lemma 5. Let $q, z_{0}, \varepsilon>0$ be as above. Set

$$
M=\left\{z:\left|z-\left(q-z_{0}\right)^{-1}\right| \leqq \varepsilon\right\} \cap\left[C-z_{0}\right]^{-1} .
$$

Let $\lambda e^{i \theta} \in M$ and $\mu>\lambda$, where $\mu$ and $\lambda$ are real. Then dist $\left[\mu e^{i \theta}\right.$, convex hull $\left.\left[C-z_{0}\right]^{-1}\right] \geqq K_{0}(\mu-\lambda)$ for some constant $K_{0}>0$, which depends only on $\left[C-z_{0}\right]$ and $\varepsilon$ and not upon the point $\lambda e^{i \theta} \in M$ selected.

Proof. By the previous lemma, we see that the tangent $S_{\theta}$ to $\left[C-z_{0}\right]^{-1}$ at $\lambda e^{i \theta}$ is a support line if $\lambda e^{i \theta} \in M$. Thus, dist $\left[\mu e^{i \theta}\right.$, convex hull $\left.\left[C-z_{0}\right]^{-1}\right] \geqq \operatorname{dist}\left[\mu e^{i \theta}, S_{\theta}\right]=(\mu-\lambda) \sin A(\theta)$, where $A(\theta)$ has the same meaning as in $\S I$. Since the tangent is a continuous function of $\theta$ and does not pass through the origin, the rest of the proof is the same as in Lemma 3.

The analogue of Lemma 5 for the expression $\operatorname{dist}\left[\mu e^{i \theta},\left[C-z_{0}\right]\right]$ is straight forward, since $C$ is both smooth and convex.

THeORem 2. Let $\sigma(T)$ lie on a smooth convex curve $C$. Then $T$ is normal if and only if

(1) $W(T) \subset \Sigma(T)$

(2) $W\left[(T-z I)^{-1}\right] \subset \Sigma\left[(T-z I)^{-1}\right]$ for $z \notin \sigma(T)$.

Proof. We first prove sufficiency. The plan of attack is the following. We will show that $T=T_{1} \oplus T_{2}$, where $T_{1}$ is normal with spectrum on an arc $\tau$, and $T_{2}$ has its spectrum on closure $[\sigma(T) \backslash \tau]$. Repeating this argument yields the proof. 
Choose $q, z_{0}, \varepsilon>0$ such that $\left[C-z_{0}\right]^{-1}$ is convex at every point of

$$
M=\left\{z:\left|z-\left(q-z_{0}\right)^{-1}\right| \leqq \varepsilon\right\} \cap\left[C-z_{0}\right]^{-1}
$$

Set $S=T-z_{0} I=B U$, and let $U=\int e^{i t} d E(t)$. This time we will consider only $y \in E(\gamma)$, where $\gamma$ is that part of the unit circle contained in the sector from $\min \arg M^{-1}$ to $\max \arg M^{-1}$

Subdivide $\gamma$ into $N$ equal parts, and define $P_{k}$ as before. Note that $P_{k} \leqq E(\gamma)$. Then using Lemma 5 and the same argument, we obtain as before $B U x=a \lambda x+a \beta w+b B y$ and $U B x=a \lambda x+b \lambda y+\beta U w$, for $\imath \in P_{k} H$, where $|b| \leqq \pi / N$ and $|\beta| \leqq K / N$ for a constant $K$ which is independent of $N$.

We will first show that $E(\gamma) H$ reduces $T$. Note that for $x \in P_{k} H$, with $\|x\|=1, S x=u+v$, where $u \in E(\gamma) H, v \in E\left(\gamma^{\prime}\right) H$ and $\|v\| \leqq$ $2 K / N$. Thus, for $y \in E(\gamma) H$, we set $y=\sum_{1}^{N} a_{k} x_{k}$, where $x_{k} \in P_{k} H$ and $\sum_{1}^{N}\left|a_{k}\right|^{2}=\|y\|^{2}$. Then $S y=\sum_{1}^{N} a_{k}\left(u_{k}+v_{k}\right)$, where $u_{k} \in E(\gamma)$ and $v_{k} \in E\left(\gamma^{\prime}\right) H$. Hence,

$$
\begin{aligned}
\left\|\sum_{1}^{N} a_{k} v_{k}\right\| & \leqq\left[\sum_{1}^{N}\left|a_{k}\right|^{2} \sum_{1}^{N}\left\|v_{k}\right\|^{2}\right]^{1 / 2} \\
& \leqq\|y\|\left[\sum_{1}^{N} 4 K^{2} / N^{2}\right]^{1 / 2} \\
& \leqq 2\|y\| K / N^{1 / 2} .
\end{aligned}
$$

Letting $N$ tend to $\infty$, this implies $S y \in E(\gamma) H$. The argument may be repeated for $S^{*}=B U^{*}$ to show that $S^{*} E(\gamma) H \subset E(\gamma) H$. One need only observe that since, for $x \in P_{k} H, U x=a x+b w$, it follows that $U^{*} x=\bar{a} x+b_{1} w_{1}$, where again $\left|b_{1}\right| \leqq \pi / N$ and all other estimates are as before.

Thus, $E(\gamma) H$ is a reducing subspace of $S$. By the estimates on $B U$ and $U B$ given above, it follows that $S$ is normal on $E(\gamma) H$. Hence $S=S_{1} \oplus S_{2}$, where $S_{1}$ is normal with $\sigma\left(S_{1}\right) \subset J=\left[C-z_{0}\right] \cap M^{-1}$, and $\sigma\left(S_{2}\right)=$ closure $[\sigma(S) \backslash J]$.

Returning to $T$, we see that $T=T_{1} \oplus T_{2}$, where $T_{1}$ is normal and $\sigma\left(T_{1}\right)$ is a segment of the arc $C$. Using the obvious compactness argument would finish the proof. However, because we have Corollary 3 in mind, we reason as follows.

Consider $T=T_{3} \oplus T_{4}$, where $T_{3}$ is normal and $T_{4}$ is completely nonnormal, i.e., normal on no reducing subspace. By the above argument $\sigma\left(T_{4}\right)=\varnothing$, and thus $T$ is normal, which completes the proof of the sufficiency. The necessity is clear.

It should be remarked that, although $\sigma(T-z I)$ is a smooth convex curve, in general $\sigma\left[(T-z I)^{-1}\right]$ is not convex. Thus $\Sigma\left[(T-z I)^{-1}\right]$ may engulf $\sigma\left[(T-z I)^{-1}\right]$ at many points. 
COROLlary 1. Let $\sigma(T)$ lie on a smooth convex curve. We list the following conditions:

A1 $R_{s p}(T-z I)=\|T-z I\|$

A2 $|W(T-z I)|=\|T-z I\|$

A3 every disc containing $\sigma(T)$ is a spectral set for $T$

A4 every disc containing $W(T)$ is a spectral set for $T$

A5 $\|(T-z I) x\| \geqq\|x\| \operatorname{dist}[z, \Sigma(T)]$

for all z above;

B1 $R_{s p}\left[(T-w I)^{-1}-z I\right]=\left\|(T-w I)^{-1}-z I\right\|$

$\mathrm{B} 2\left|W\left[(T-w I)^{-1}-z I\right]\right|=\left\|(T-w I)^{-1}-z I\right\|$

B3 every disc containing $\sigma\left[(T-w I)^{-1}\right]$ is a spectral set for $(T-w I)^{-1}$

B4 every disc containing $w\left[(T-w I)^{-1}\right]$ is a spectral set for $(T-w I)^{-1}$

$\mathrm{B} 5 \quad\left\|\left[(T-w I)^{-1}-z I\right] x\right\| \geqq\|x\| \operatorname{dist}\left[z, \Sigma\left[(T-w I)^{-1}\right]\right]$ for $w \in \sigma(T)$ and all $z$ above.

Given one condition from column $A$ and one from column $B$, then $T$ is normal. Conversely, if $T$ is normal, then all conditions under $A$ and $B$ hold.

Proof. Each condition in column A implies $W(T) \subset \Sigma(T)$ (see [11] for 1-4 and [6] for 5). Each condition in column $B$ implies $W\left[(T-w I)^{-1}\right] \subset \Sigma\left[(T-w I)^{-1}\right]$ for $w \notin \sigma(T)$. This completes the proof.

COROLlary 2. If $T$ is hyponormal and $\sigma(T)$ lies on a smooth convex curve, then $T$ is normal.

Proof. If $T$ is hyponormal, then $(T-w I)^{-1}$ is hyponormal for $w \notin \sigma(T)$. Thus $T$ satisfies $\mathrm{A} 1$ and $\mathrm{B} 1$ (see [9]).

This result has been obtained by both Putnam [7] and the author [9]. In fact, both papers prove somewhat more general theorems. However, in all three cases, the method of proof is radically different. Moreover, no one approach covers all cases where normality is known to hold.

COROLLARY 3. In Theorem 2 and both corollaries, one can replace $\sigma(T)$ on a smooth convex curve by $\sigma(T)$ on a piecewise smooth convex curve.

Proof. By the techniques of Theorem 2, it can be shown that $T=T_{1} \oplus T_{2}\left(\right.$ on $\left.H=H_{1} \oplus H_{2}\right)$, where $T_{1}$ is normal, and $\sigma\left(T_{2}\right)$ consists 
of a finite number of points. It is not hard to show that $T_{2}$ is also normal. Indeed, since $\sigma\left(T_{2}\right)$ is a finite set of points, $T_{2}$ is spectral. The condition $W\left[(T-z I)^{ \pm 1}\right] \subset \Sigma\left[(T-z I)^{ \pm 1}\right]$ for $z \notin \sigma(T)$ leads to the growth condition $\left\|(T-z I)^{-1}\right\| \leqq M / \operatorname{dist}[z, \sigma(T)]$, for some constant $M$. This implies $T_{2}$ is scalar (see [1] or [3]). Hence $T_{2}$ has a complete set of eigenvectors which span $H_{2}$. By the argument from the finite dimensional case, the eigenvectors are orthogonal, which completes the proof.

REMARK. In Theorem 2, the condition

$$
W\left[(T-z I)^{-1}\right] \subset \Sigma\left[(T-z I)^{-1}\right] \quad \text { for } z \notin \sigma(T)
$$

may be weakened to

$$
W\left[(T-z I)^{-1}\right] \subset \Sigma\left[(T-z I)^{-1}\right] \quad \text { for } z \in U,
$$

and $z \in \sigma(T)$, where $U$ is any open set containing $\sigma(T)$.

It is well known that there is a close connection between the numerical range and the rate of growth of the resolvent. Indeed, $W(T) \subset \Sigma(T)$ if and only if $\left\|(T-z I)^{-1}\right\| \leqq 1 / \operatorname{dist}[z, \Sigma(T)]$ for all $z \notin \Sigma(T)$, (see [6]).

This leads us to the following conjecture. Let $\sigma(T)$ lie on a smooth convex curve. Then $T$ is normal if and only if

$$
\left\|(T-z I)^{-1}\right\| \leqq 1 / \operatorname{dist}[z, \sigma(T)] \quad \text { for } z \notin \sigma(T) .
$$

This is known to be true if $\sigma(T)$ is a line segment [5], or if $\sigma(T)$ is a circle [2].

Added in proof: The hypotheses of Theorem 1 may be weakened from $W\left(T^{ \pm 1}\right) \subset \Sigma\left(T^{ \pm 1}\right)$ to $W\left(T^{ \pm 1}\right) \subset$ convex hull $\left(C^{ \pm 1}\right)$ where $\sigma(T)$ lies on $C$. This may be seen in two ways. First, the proof uses only the weaker assumption. Second, let $S=T \oplus A$, where $A$ is normal and $\sigma(A)=C$. Then, Theorem 1 , as it stands, implies $S$ is normal, and thus, so is $T$.

\section{REFERENCES}

1. R. G. Bartle, Spectral localization of operators in Banach space, Math. Annalen 153 (1964), 261-269.

2. W. F. Donoghue, On a problem of Nieminen, Inst. Hautes Etudes Sci. Publ. Math. 16 (1963), 127-129.

3. N. Dunford, Spectral operators, Pacific J. Math. 4 (1954), 321-354.

4. C. H. Meng, On the numerical range of an operator, Proc. Amer. Math. Soc. 14 (1963), 167-171.

5. T. Nieminen, $A$ condition for the self-adjointness of a linear operator, Ann. Acad. 
Sci. Fenn. Ser. A. I, No. 316 (1962), 3-5.

6. G. Orland, On a class of operators, Proc. Amer. Math. Soc. 15 (1964) 75-80.

7. C. R. Putnam, On the spectra of semi-normal operators, Trans. Amer. Math. Soc. 119 (1965), 509-523.

8. M. Schreiber, Numerical range and spectral sets, Michigan Math. J. 10 (1963), 283-288.

9. J. G. Stampfli, Hyponormal operators and spectral density, Trans. Amer. Math. Soc. 117 (1965), 469-476.

10. F. A. Valentine, Convex Sets, McGraw-Hill, New York, 1964.

11. J. Williams, Spectral sets and finite dimensional operators, Thesis, University of Michigan 1965.

Received October 17, 1966. This work was partially supported by the National Science Foundation, under Grant No. GP-5152. Some of the results of this paper were previously announced in the Bull. Amer. Math. Soc., Nov. 1966.

NEW YORK UNIVERSITY 


\section{PACIFIC JOURNAL OF MATHEMATICS}

\section{H. ROYDEN}

Stanford University

Stanford, California

\author{
J. P. JANS \\ University of Washington \\ Seattle, Washington 98105
}

\section{EDITORS}

\author{
J. DugundJI \\ Department of Mathematics \\ Rice University \\ Houston, Texas 77001 \\ Richard ARENS \\ University of California \\ Los Angeles, California 90024
}

\section{ASSOCIATE EDITORS}
E. F. BECKENBACH
B. H. NeumanN
F. WOLF
K. YOSIDA

\section{SUPPORTING INSTITUTIONS}

\author{
UNIVERSITY OF BRITISH COLUMBIA \\ CALIFORNIA INSTITUTE OF TECHNOLOGY \\ UNIVERSITY OF CALIFORNIA \\ MONTANA STATE UNIVERSITY \\ UNIVERSITY OF NEVADA \\ NEW MEXICO STATE UNIVERSITY \\ OREGON STATE UNIVERSITY \\ UNIVERSITY OF OREGON \\ OSAKA UNIVERSITY \\ UNIVERSITY OF SOUTHERN CALIFORNIA
}

\author{
STANFORD UNIVERSITY \\ UNIVERSITY OF TOKYO \\ UNIVERSITY OF UTAH \\ WASHINGTON STATE UNIVERSITY \\ UNIVERSITY OF WASHINGTON \\ AMERICAN MATHEMATICAL SOCIETY \\ CHEVRON RESEARCH CORPORATION \\ TRW SYSTEMS \\ NAVAL ORDNANCE TEST STATION
}

Mathematical papers intended for publication in the Pacific Journal of Mathematics should be typewritten (double spaced). The first paragraph or two must be capable of being used separately as a synopsis of the entire paper. It should not contain references to the bibliography. Manuscripts may be sent to any one of the four editors. All other communications to the editors should be addressed to the managing editor, Richard Arens at the University of California, Los Angeles, California 90024.

50 reprints per author of each article are furnished free of charge; additional copies may be obtained at cost in multiples of 50 .

The Pacific Journal of Mathematics is published monthly. Effective with Volume 16 the price per volume (3 numbers) is $\$ 8.00$; single issues, $\$ 3.00$. Special price for current issues to individual faculty members of supporting institutions and to individual members of the American Mathematical Society: $\$ 4.00$ per volume; single issues $\$ 1.50$. Back numbers are available.

Subscriptions, orders for back numbers, and changes of address should be sent to Pacific Journal of Mathematics, 103 Highland Boulevard, Berkeley 8, California.

Printed at Kokusai Bunken Insatsusha (International Academic Printing Co., Ltd.), 7-17, Fujimi 2-chome, Chiyoda-ku, Tokyo, Japan.

PUBLISHED BY PACIFIC JOURNAL OF MATHEMATICS, A NON-PROFIT CORPORATION

The Supporting Institutions listed above contribute to the cost of publication of this Journal, but they are not owners or publishers and have no responsibility for its content or policies. 


\section{Pacific Journal of Mathematics}

A. A. Aucoin, Diophantine systems ............................. 419

Charles Ballantine, Products of positive definite matrices. I ............... 427

David Wilmot Barnette, A necessary condition for d-polyhedrality ............ 435

James Clark Beidleman and Tae Kun Seo, Generalized Frattini subgroups of finite groups ......................................... 441

Carlos Jorge Do Rego Borges, A study of multivalued functions ............. 451

William Edwin Clark, Algebras of global dimension one with a finite ideal

lattice ...............................................

Richard Brian Darst, On a theorem of Nikodym with applications to weak convergence and von Neumann algebras .........................

George Wesley Day, Superatomic Boolean algebras .....................

Lawrence Fearnley, Characterization of the continuous images of all

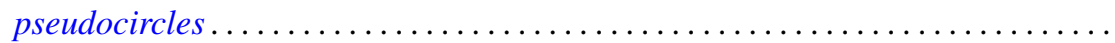

Neil Robert Gray, Unstable points in the hyperspace of connected subsets....... 515

Franklin Haimo, Polynomials in central endomorphisms .................. 521

John Sollion Hsia, Integral equivalence of vectors over local modular lattices . . . . 527

Jim Humphreys, Existence of Levi factors in certain algebraic groups .......... 543

E. Christopher Lance, Automorphisms of postliminal $C^{*}$-algebras ............ 547

Sibe Mardesic, Images of ordered compacta are locally peripherally metric . . . . 557

Albert W. Marshall, David William Walkup and Roger Jean-Baptiste Robert Wets,

Order-preserving functions: Applications to majorization and order

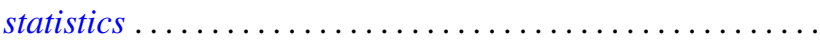

Wellington Ham Ow, An extremal length criterion for the parabolicity of

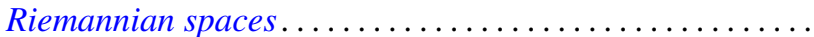

585

Wellington Ham Ow, Criteria for zero capacity of ideal boundary components of

Riemannian spaces...................................... 591

J. H. Reed, Inverse limits of indecomposable continua .................. 597

Joseph Gail Stampfli, Minimal range theorems for operators with thin spectra . . . 601

Roy Westwick, Transformations on tensor spaces..................... 613

Howard Henry Wicke, The regular open continuous images of complete metric

spaces ........................................... 621

Abraham Zaks, A note on semi-primary hereditary rings .............. 627

Thomas William Hungerford, Correction to: "A description of $\operatorname{Mult}_{i}\left(A^{1}, \cdots, A^{n}\right)$

by generators and relations" ............................. 629

Uppuluri V. Ramamohana Rao, Correction to: "On a stronger version of Wallis' formula".............................................. 629

Takesi Isiwata, Correction: "Mappings and spaces" ................... 630

Henry B. Mann, Josephine Mitchell and Lowell Schoenfeld, Correction to:

"Properties of differential forms in $n$ real variables" .... . .

James Calvert, Correction to: "An integral inequality with applications to the

Dirichlet problem"............................. 